\title{
Abordaje fonoaudiológico
} de la deglución en

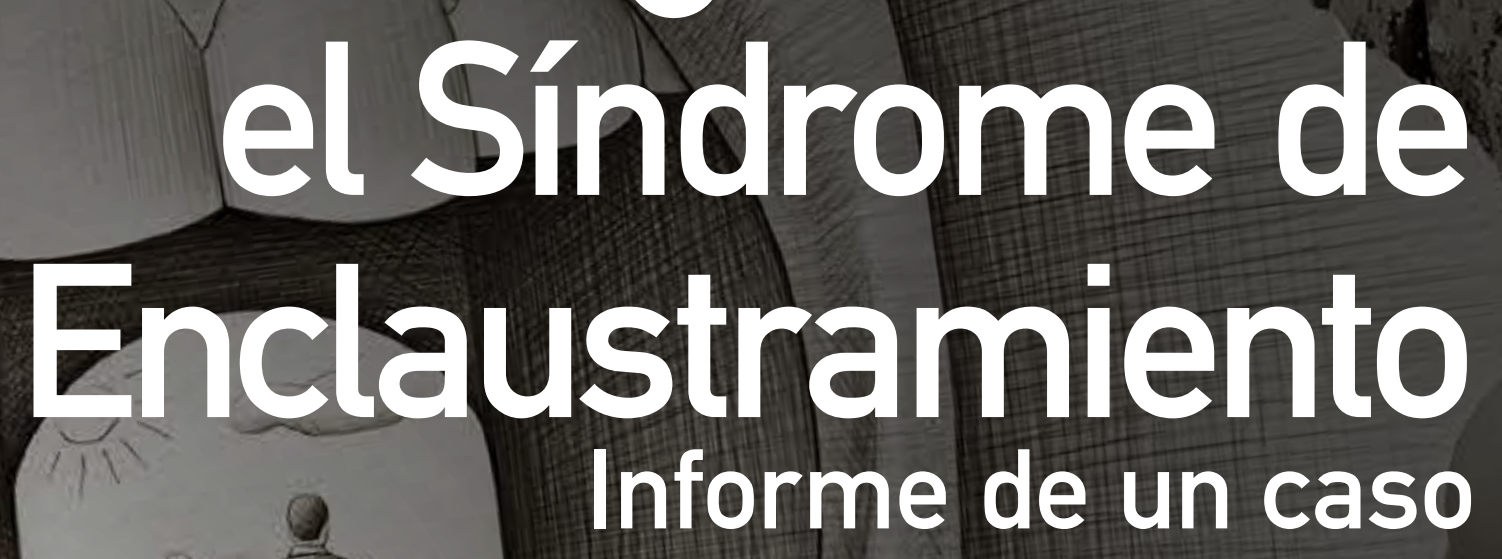

Intervention approach for speech -language pathologists to swallowing in Locked-in Syndrome : A case report

(c) $\frac{10(0)}{\mathrm{Br} N \mathrm{Na}}$
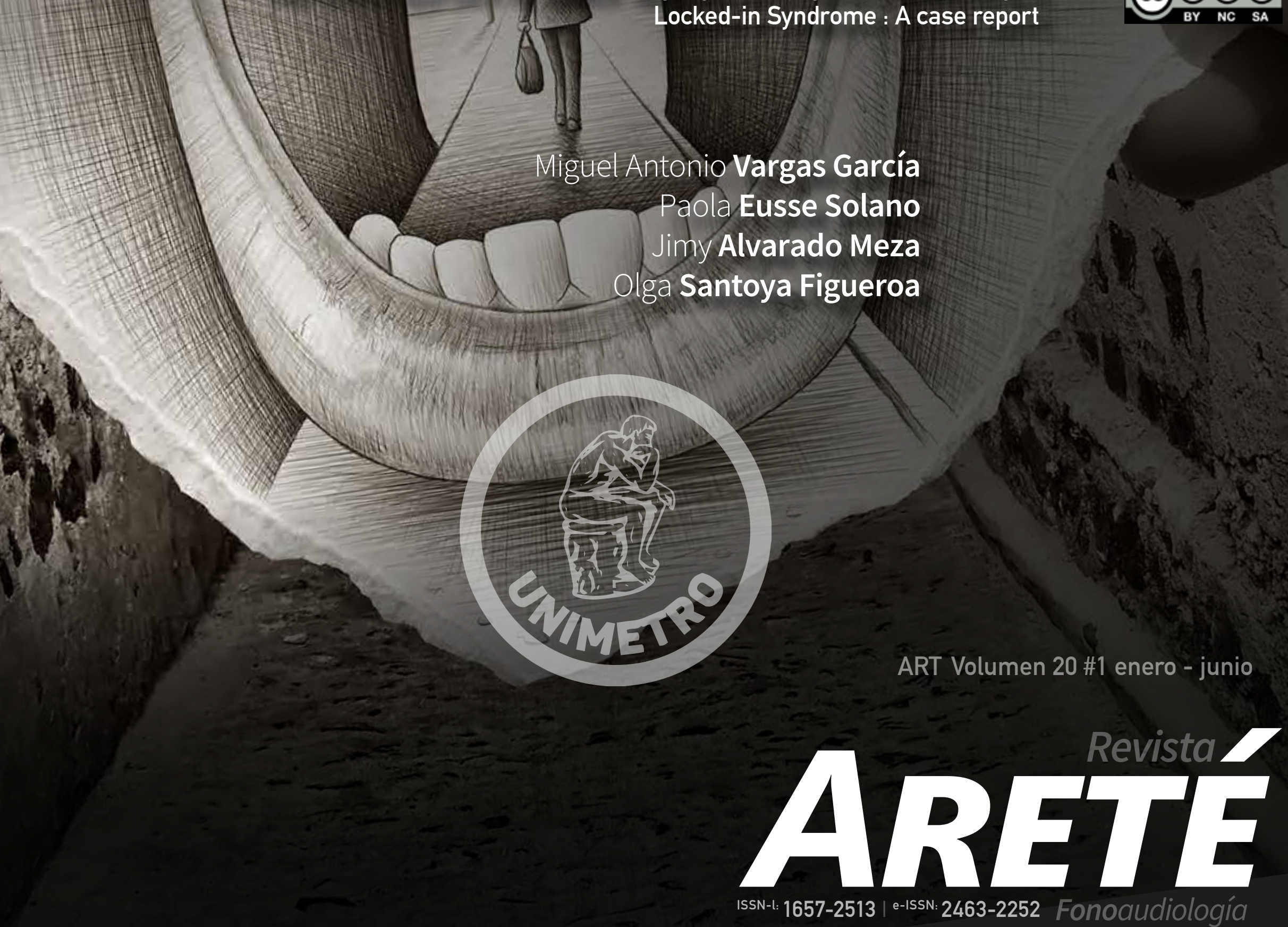
ARETÉ

1657-2513 | e-15SN: 2463-2252 Fonoaudiología

ID:

1657-2513.art.20104

Title: Intervention approach for speech -language pathologists to swallowing in Locked-in Syndrome

Subtitle: A case report

Título: $\quad$ Abordaje fonoaudiológico de la deglución en el Síndrome de Enclaustramiento

Subtítulo: $\quad$ Informe de un caso

Alt Title / Título alternativo:

[en]: $\quad$ Abordaje fonoaudiológico de la deglución en el Síndrome de Enclaustramiento

[es]: $\quad$ Abordaje fonoaudiológico de la deglución en el Síndrome de Enclaustramiento

Author (s) / Autor (es):

Vargas García, Eusse Solano , Alvarado Meza \& Santoya Figueroa

Keywords / Palabras Clave:

[en]: $\quad$ Locked-in syndrome; Deglutition; Speech, Language pathologists and Hearing Sciences; Case Reports; Deglutition Disorders; Therapeutics

[es]: $\quad$ Síndrome de Enclaustramiento; Deglución; Fonoaudiología; Informes de caso; Trastornos de Deglución; Terapéutica

Financiación / Funding:

Fonoaudiología Deglución

Submited: $\quad$ 2020-01-20

Acepted: 2020-02-19
Resumen

En el Síndrome de Enclaustramiento las manifestaciones clínicas son preponderantemente de orden motor, situación que requiere la intervención del Fonoaudiólogo, dado el compromiso comunicativo y de la biomecánica deglutoria. La disfagia en este síndrome está dada por la desconexión sensorio-motriz de las estructuras anatómicas de la deglución, alterando la secuencia neuromotora y sensitiva del proceso deglutorio debido a la desconexión pontina. El texto presenta el caso de una paciente femenina de 27 años quien es diagnosticada con Síndrome

de Eclaustramiento posterior a accidente cerebrovascular de Arteria Basilar Derecha, acto que compromete sus habilidades motoras de forma generalizada. A partir de su diagnóstico y dadas las características multicéntricas de la deglución, se objetiva comenzar intervención

fonoaudiológica oportuna, estableciendo como eje fundamental la secuencialidad de los procesos neurofisiológicos de la deglución; adoptando como estrategia el manejo de consistencias, fortaleciendo sensorial y motrizmente la deglución. Al intervenir las necesidades sensoriomotoras orales en pro de la alimentación, y usando como estrategias terapéuticas la modificación de consistencias, manejo de volumen, postura, aditamento para suministro de alimento y tiempos de ingesta, se minimizan

los riesgos en el proceso de alimentación y se puedo llegar a mantener un estado nutricional y de hidratación óptimo para la recuperación física general, permitiendo el aumento de la ingesta en una proporción inicial de 30\% vía oral - 70\%

vía enteral a un 100\% incluyendo a la dieta el

nivel 4-puré, según la IDDSI y desarrollando paralelamente procesos de interacción familiar, impactando su calidad de vida.

\section{Citar como:}

Vargas García, M. A., Eusse Solano , P. ., Alvarado Meza , J. \& Santoya Figueroa , O. . (2020). Abordaje fonoaudiológico de la deglución en el Síndrome de Enclaustramiento: Informe de un caso. Areté, 29-37. Obtenido de: https://arete.ibero.edu.co/article/view/1762

\section{Abstract}

The clinical manifestations of Locked-In Syndrome are predominantly motor in order, a situation that requires the intervention of the speech therapist, given the communicative disorder and biomechanics of swallowing. The dysphagia in this syndrome is produced by the sensibly-motor disconnection of the anatomical structures of swallowing, altering the neuromotor and sensitive sequence of the swallowing process because of the pontine disconnection. The text presents the case of a 27-year-old female who is diagnosed with Locked-In Syndrome after suffering a cerebrovascular accident in the Basilar Right

Artery, which compromises her motor skills. From her diagnosis and given the characteristics of swallowing, it is necessary to begin speech therapy intervention, focusing the sequences of the neurophysiological processes of swallowing; using as a strategy the management of consistency, strengthening sensory and motor swallowing. After intervening the oral sensory motor, improving

nutrition, and using as therapeutic strategies the modification of consistency, volume, posture, food supply and intake times. The risks in the feeding process are minimized, the nutritional status and optimal hydration can be maintained for the overall physical recovery, allowing the increase of intake with an initial proportion of 30\% orally - 70\% through enteral to 100\%, including the 4-Pureed level, according to the IDDSI and developing parallel processes of family interaction, impacting their quality of life.

\begin{abstract}
Miguel Antonio Vargas García , Dr MsC $\mathrm{BHS}$
\end{abstract}

\section{ORCID: $\quad$ 0000-0002-5639-9474}

\section{Source | Filiacion:}

Universidad Metropolitana de BarranquiIla

BIO:

Doctor en Fonoaudiología, Magister en Educación, Especialista en Terapia Miofuncional

\section{City | Ciudad:}

Barranquilla [co]
Paola Eusse Solano, sp

\section{Source | Filiacion:}

Universidad Metropolitana de BarranquiIla

BIO:

Fonoaudióloga especialista en Docencia Universitaria

City | Ciudad:

Barranquilla [co]
Jimy Alvarado Meza, MSc BHS

\section{Source | Filiacion:}

Universidad Metropolitana de BarranquiIla

BIO:

Fonoaudiólogo. Magíster en Disturbios de de la Comunicación Humana con Énfasis en Deglución y Disfagia. Docente Investigador Universidad Metropolitana

\section{City | Ciudad:}

Barranquilla [co]
Olga Santoya Figueroa, BHS sp

\section{Source | Filiacion:}

Universidad Metropolitana de BarranquiIla

BIO:

Fonoaudióloga especialista en Audiología

\section{City | Ciudad:}

Barranquilla [co] 


\section{Abordaje fonoaudiológico de la deglución en el Síndrome de

Intervention approach for speech -language pathologists to swallowing in Locked-in Syndrome : A case report

Miguel Antonio Vargas García

Paola Eusse Solano

Jimy Alvarado Meza

Olga Santoya Figueroa

\section{Introducción}

Al iniciar el tratamiento terapéutico en casos sindrómicos y no sindrómicos, se hace necesario que el equipo profesional tratante tenga claridad acerca de los componentes circundantes al diagnóstico inicial, generando un cuadro específico de los factores desarrollados a partir de cada enfermedad.

Al hablar del abordaje clínico de un síndrome, el profesional de salud debe comprender que todas las manifestaciones del paciente estarán dadas por diversas condiciones asociadas a particularidades anatómicas, fisiológicas o bioquímicas que condiciona la alteración y repercuten en errores sistémicos o no sistémicos que definen la enfermedad, sus signos y síntomas (Biblioteca Virtual de Salud, 2018).

Después de un proceso analítico, generado a través de un razonamiento científico y de hilar las diferentes manifestaciones clínicas del paciente y su relación en los procesos funcionales y el desenvolvimiento personal y contextual, se deben definir los profesionales facultados en la atención de las distintas características presentes, para favorecer no sólo aspectos biológicos contemplados en la salud, sino también aspectos sociales y emocionales fundamentados en la calidad de vida (Vargas M. , 2017) (Vargas M. , 2018). 
El Síndrome de Enclaustramiento, LIS por sus siglas en inglés, ha sido definido como una condición de muy baja prevalencia, en el cual, quien la padece se encuentra encerrado en su propio cuerpo, con un estado de conciencia y cognitivo óptimo, pero un estado vegetativo a nivel motor (Pacheco-Hernández, y otros, 2017) (Vidal, 2018). Está codificado en la Clasificación Internacional de Enfermedades-Décima edición (CIE-10) como $\mathbf{G 8 3 . 5}$ - también conocido como Síndrome de Desconexión Pontina, Síndrome de Encerramiento o Síndrome de Vigilia sin respuesta. Las manifestaciones clínicas pueden parecer claramente identificables, aunque en algunas ocasiones puede llegar a ser confundido con un estado de coma; aun así, su alteración preponderantemente de orden motor condiciona de inmediato las actuaciones clínicas de diferentes profesionales como el Fonoaudiólogo, dado el compromiso comunicativo y deglutorio.

El LIS, es una patología poco frecuente, no heredable, de origen vascular o por traumas al tronco cerebral, la arteria basilar o los pedúnculos cerebrales, corticoespinal o corticopontinas que interrumpen la conexión corticobulbar (Khanna, Verma, \& Richard, 2011); en Europa, ha sido catalogada como una enfermedad huérfana y clasificada por ORPHA bajo el código $\mathbf{2 4 0 6}$ como una enfermedad neurológica rara (Orphadata, 2018), con una incidencia de 1/1.000.000.

Su cuadro clínico evidencia tetraplejia, parálisis de nervios craneanos bajos: anartria, diplejía facial, rigidez de descerebración. Sin embargo, presenta completamente conservada la conciencia y la vigilia y en algunos casos la apertura y cierre de párpados y el movimiento vertical del globo ocular (Lujan Ramos, Monterrosa-Salazar, \& PoloVerbel, 2011).

Según (Khanna, Verma, \& Richard, 2011) el LIS presenta:

...(1) apertura sostenida del ojo (bilateral la ptosis debe descartarse como un factor de complicación); (2) preservado habilidades cognitivas básicas; (3) afonía o hipofonía severa; (4) tetraplejia o cuadriparesia; y (5) un modo primario de comunicación que usa el movimiento ocular vertical o lateral o parpadeando en el párpado superior. LIS se puede subdividir en función de la extensión del deterioro motor: (1) inmovilidad total de LIS clásica, excepto para movimientos verticales oculares o parpadeantes; (2) incompleta LIS con restos de movimientos voluntarios; (3) inmovilidad completa de LIS, que incluye todos los movimientos oculares, combinados con la conciencia conservada (p.96).

\section{Sus características se supeditan:}

La etiología, vascular o no vascular;

La fase en la que se encuentre el síndrome, aguda o crónica; La severidad, determinada como completa, incompleta o total;

Sus características de evolución, transitorias o permanentes.

Dentro de las características manifiestas del síndrome, se presentan factores que comprometen actividades ligadas a la atención fonoaudiológica de la anartria y al no control de musculatura facial; problemas de deglución y voz, lo que predispone errores en la alimentación por vía oral.

La disfagia en el LIS está dada por la desconexión sensorial y motriz de las estructuras anatómicas de la deglución, la parálisis facial, faríngea, lingual y laríngea, al igual que un compromiso de los procesos respiratorios, lo que genera un alto riesgo de penetración y/o aspiración. En una serie de 50 pacientes crónicos con LIS, el $\mathbf{7 5 \%}$ tuvo dificultad para tragar las secreciones orofaríngeas, lo que define un alto porcentaje de repercusiones deglutorias propias del quehacer del fonoaudiólogo. Sumado a la presencia de sialorrea y dificultades respiratorias (Khanna, Verma, \& Richard, 2011).

Este síndrome ha sido catalogado como catastrófico e irremediable y con pocas esperanzas en la recuperación de las habilidades perdidas, considerando el tratamiento como actividades paliativas y mantenimiento, no de rehabilitación. Sin embargo, se ha descrito evolución en diferentes casos, atribuida al pronto establecimiento de la terapéutica (Arroyo-Aljaro, González-Viejo, \& Rovira, 2008), las cuales fomentan desde la fonoaudiología aspectos de mejoría clínica, basados en acciones comunicativas y deglutorias, que impactan en la salud a nivel integral, incluyendo su desenvolvimiento social, de ahí, la importancia y el impacto de presentación de la experiencia en la atención clínica del caso, aportando a la clínica y la rehabilitación fonoaudiológica del LIS especialmente en los aspectos del trastorno deglutorio.

\section{Presentación del caso}

Paciente femenino de 27 años, sin antecedentes neurológicos relevantes; ingresa a urgencias en estado POST-ICTAL por convulsión tónico-clónica generalizada, con pérdida de conciencia, sialorrea, patrón respiratorio irregular, tensión arterial 100/55, afebril.

Es intubada con tubo orotraqueal No.8, ventilación mecánica, con ventilador Syno Vent E5, no se observa respuesta al llamado. Pasa a Unidad de Cuidados Intensivos, se indica manejo con Ácido Valproico y Benzodiacepina, en estado de coma bajo efectos de sedación, analgesia y relajación muscular, sonda nasogástrica y vesical, con sospecha de meningitis.

A las 24 horas de evolución, responde al llamado de estímulo intenso con apertura ocular; sin respuesta motriz, comunicación eficiente ni seguimiento de órdenes simples, cuadriplejía.

Se encuentra Tomografía Axial Computarizada de cráneo simple con imagen hipodensa en mesencéfalo, sugiriendo realizar resonancia, la cual confirma isquemia en región de protuberancia, realizando posteriormente panangiografía que confirma oclusión de arteria vertebral izquierda con disección y trombo en nivel de C4-C6, y oclusión en arteria basilar a nivel proximal, definiendo diagnóstico de LIS.

Se realiza angioplastia de vasos intracraneanos con colocación de stent y trombólisis mecánica con Stent Retriver. Además, traqueostomía en el noveno día de hospitalización y colocación de sonda de gastrostomía al décimo. En su día 20 de evolución, la paciente es trasladada a un centro de atención médica, con soporte de oxígeno. Hasta el momento solo ha recibido intervención por terapia física.

El diagnóstico de lesión pontina, agrede directamente el comando neural de la deglución, (nervio trigémino-V, nervio facial-VII, nervio glosofaríngeo-IX, nervio vago-X, nervio accesorio-XI y nervio hipogloso-XII), por ello el abordaje valorativo del fonoaudiólogo se hizo necesario. Se inicia abordaje clínico por fonoaudiología para definir daños funcionales de secuencialidad deglutoria y promover plan terapéutico que potencialice cualquier acción motora en la evolución del síndrome.

La valoración por fonoaudiología se dirige a la secuencialidad y funcionalidad de procesos neuromotores de la deglución, desde el concepto valvular y siguiendo el Protocolo de Evaluación Miofuncional MBGR (Flores, Berretin-Felix, Beltrati, \& Queiroz, 2009), como una herramienta que recolecta información clínica del paciente y fomenta 
la evaluación integral de aspectos anatómicos y funcionales de la región cráneo-facial-cervical. Se procede con la ejecución de una evaluación sistémica de las estructuras del complejo estomatognático.

Se encuentra un marcado imbalance muscular facial y cervical, aumento de tono especialmente en hemicuerpo derecho; condición que dificulta el control cefálico, generando desviación de la cabeza hacia izquierda, sin seguimiento de mirada.

Los comandos motores faciales se encuentran alterados, extensión de los movimientos disminuida y fuerza muscular disfuncional, con mayor compromiso de hemicara izquierda. Esta condición isométrica fomenta la desviación de comisura labial hacia lado afectado, ocasionando selle labial incompleto. Actividades motoras como la sonrisa, permite el desplazamiento de las comisuras con mayor simetría en situaciones espontáneas, mediadas por agentes externos que facilitaban la extensión y precisión del movimiento.

Se encuentra lengua dentro de cavidad oral, con fasciculaciones, realización de movimientos repetitivos y espontáneos para deglución de saliva, protrusión disminuida y sin ejecución de movimientos laterales y anteroposteriores coordinados. Se evidencia latencia del reflejo deglutorio ante el manejo del control salival. Se evalúa reflejo gag con estimulación táctil, mostrándose posteriorizado y disminuido; el reflejo de tos se presenta de manera espontánea pero débil, lo que supone inefectividad para el proceso de protección, aumentando factores de riesgo para penetraciones y aspiraciones.

La falta de coordinación en la secuencialidad de los patrones motores orofaciales y las respuestas ante la exploración sensorial en la paciente, evidencian compromisos para la eficacia deglutoria, desencadenados por la desconexión neural, condición que ha generado dificultades en la secuencia motora y la recepción sensorial general.

Hasta el inicio de la evaluación fonoaudiológica, por orden médica no se recibe alimentos ni medicación por vía oral, encontrando paciente en un nivel 1 según la Escala Funcional de Ingesta por vía oral FOIS (Furkim \& Freitas, 2008), entendida como una escala que describe la realidad del paciente con disfagia en función a la habilitación de la vía oral y de las consistencias utilizadas.

Dadas las características de manejo de saliva, se trabaja ganancia propioceptiva oral para incrementar la receptividad de los actores dinámicos de la deglución, luego se planea la ejecución del Blue Dye Test como indicador de aspiraciones. Este se considera un procedimiento simple y económico para la predicción de aspiraciones en el paciente traqueostomizado, tomando como positiva la presencia de coloración azul en cualquier lugar de la traqueostomía o durante el procedimiento de aspiración traqueal; la literatura le ha atribuido entre un $\mathbf{8 5 \%}$ y un $\mathbf{1 0 0} \%$ de sensibilidad en casos de ventilación mecánica, $\mathbf{7 6} \%$ en casos no ventilados y $\mathbf{1 0 0} \%$ de especificidad (Belafsky, Blumenfeld, LePage, \& Nahrstedt, 2003) (Simao, y otros, 2009) (Fiorelly, y otros, 2017)

Pasados 8 días de la implementación del plan terapéutico propioceptivo, el cual contenía ejercicios de alcance y movilidad lingual y palatal, se encuentra paciente en posición habitual, decúbito dorsal, alerta, consciente, respondiendo al llamado por su nombre, con un sistema comunicativo alternativo basado en movimientos oculares y palpebrales. Como prueba para verificar la aspiración en pacientes traqueostomizados (Fiorelly, y otros, 2017), se procede a realizar el Blue Dye Test, modificado, utilizando agua en consistencia pudín y teñido con anilina vegetal; el resultado evidenció latencia en el desencadenamiento de la deglución ante una orden verbal y la necesidad de degluciones múltiples para la ingesta en los siguientes volúmenes: 5, 10 y $15 \mathrm{ml}$, presentando mayor control de cada una de las consistencias ofertadas hasta $10 \mathrm{ml}$, con inadecuado manejo de las mismas en $15 \mathrm{ml}$, observándose residuos en cavidad oral.

Pasados 15, 30 y 60 minutos después de realizado el procedimiento, se hace verificación de la cánula de traqueostomía y secreciones, definiendo un resultado de Blue Dye Test negativo, lo que permite generar inferencias acerca de la funcionalidad de la protección aérea durante la deglución faríngea. En consecuencia, se hace remisión a Videofluroscopia para evaluación con consistencias y volúmenes, y se establece un trabajo detallado, sensoriomotor en las válvulas deglutorias. Se precisa valoración instrumental 30 días calendario después de la obtención del resultado, tiempo estimado para la ejecución pasiva y activa de un plan terapéutico miofuncional.

Con el objetivo de evidenciar de manera directa el comportamiento de la protección de vía aérea inferior, se realiza como como estrategia radiológica la Videofluroscopia de la deglución (Barros, Silva, \& De Angelis, 2010), un mes después de iniciar el proceso terapéutico. El procedimiento se ejecuta con consistencia líquida clara, néctar, miel y pudín. Los resultados evidencian un diagnóstico de Disfagia Orofaríngea leve a moderada, Nivel 4 (O’Neil, Purdy, Falk, \& Gallo, 1999), con clasificación en escala de penetración/aspiración nivel 2, la cual corresponde a que se encuentra contraste encima de las cuerdas vocales (Rosenbek, Robbins, Roecker, Coyle, \& Wood, 1996), manifestando una fase oral con captación adecuada del alimento sin escape anterior, mayor coordinación en fase oral preparatoria, eficaz en el manejo del bolo alimenticio para todas las consistencias, movilidad anteroposterior de lengua eficiente para propulsión del bolo a orofaringe, aunque con fuerza disminuida. No se observó escape prematuro a orofaringe, ni degluciones múltiples. Masticación lentificada pero eficiente para las consistencias ofertadas. Sin presencia de estasis a nivel intraoral.

En cuanto a la fase faríngea, se observa competencia velar y un contacto adecuado de base de lengua y pared posterior de la faringe; presencia de residuos de alimento pos-deglución en vallécula en consistencia pudín, la cual se limpia con la adopción de la deglución en seco como estrategia final de limpieza. De igual manera, se evidencia penetración en consistencia miel, néctar y líquido, la cual limpia generando una segunda deglución. No se produce tos antes, durante, después de la ingesta. No se ejecuta evaluación videofluroscópica en fase esofágica.

Dado los resultados encontrados, Fonoaudiología propone al equipo médico tratante y a la familia, un protocolo de habilitación de vía oral en proporciones mínimas, fluctuando con una mayor proporción por vía alternativa presente, aspecto que pretende activar la funcionalidad de cavidad oral-faríngea y sumar indicadores de calidad de vida, devolver a la paciente el placer del alimento en boca y a la familia indicadores de evolución contrastables. Se define entonces un nivel 2 según la FOIS.

A partir de los resultados obtenidos y con la caracterización hecha en la evaluación fonoaudiológica, se inicia proceso de intervención con actividades terapéuticas pasivas y activas, enfocadas a favorecer los niveles de sensibilidad e información propioceptiva orofacial, además de generar bases para establecer patrones neuromusculares funcionales de la acción oral faríngea.

El desarrollo de las actividades está encaminado al uso de temperaturas, texturas, estimulación neurosensorial en cabeza y cuello, movilización de tejidos blandos a nivel facial, oral e intraoral. Paralelamente se realiza un plan con ejercicios que coadyuven a la funcionalidad de las estructuras trabajadas, enfocando el esfuerzo en la amplitud de movimientos linguales, labiales y palatales, con el objeto de favorecer la actividad propioceptiva, de control y manejo del bolo 


\section{Abordaje fonoaudiológico de la deglución en el Síndrome de Enclaustramiento}

Informe de un caso

alimenticio en cavidad oral, dinamizando la secuencia funcional de la deglución en esta cavidad (Guevara, Avendaño, Salazar, Alarcón, \& Santelices, 2016).

Por último, se plantea un trabajo de reconocimiento y manejo sensorial de consistencias, como estrategia para potencializar la vía oral (Steele, $y$ otros, 2015). Se maneja la estrategia con base en lo propuesto por la iniciativa internacional de estandarización de dieta para la disfagia IDDSI (International Dysphagia Diet Standardisation Initiative, 2018).

Una vez se inicia proceso de intervención, se registra diariamente la consecución de objetivos por sesión, con el fin de detectar los posibles avances. Se observa un mayor control y funcionalidad de las estructuras estomatognáticas; la primera verificación de avances, pasadas 20 sesiones, se estableció en funcionalidad lingual, que había sido llevada junto a acciones isotónicas e isométrica a la mejora de la extensión y el alcance de movimientos.

La estimulación propioceptiva y motora ejercida en la estructura lingual, permitió que la paciente hiciera uso de movimientos linguales en distintos planos; los movimientos se generaban de manera incoordinada pero cada vez con mayor extensión. Se asociaban tremores al movimiento, pero se lograba ejecutar acciones de protrusión y lateralización, cada vez con mayor facilidad y coordinación. El mejor desempeño se materializaba siempre hacia lado derecho. Una vez logradas acciones de movilidad lingual, se ejercita de forma pasiva acciones de elevación de ápice lingual con el fin de proyectar el movimiento funcional de la lengua en ingesta por vía oral y comenzar la estrategia terapéutica que diera forma al objetivo de habilitación de la vía oral de alimentación (Pereira, Carrera, \& Brandão, 2010).

Con la lengua como apoyo inicial, pasadas 35 sesiones, y con resultados motores y propioceptivos intraorales favorables, se encamina la intervención al manejo y deglución de la alimentación. Se establece como estrategia inicial la IDDSI (International Dysphagia Diet Standardisation Initiative, 2016) con la utilización de la consistencia moderadamente espesa; dadas las características de maniobrabilidad oral, la consistencia moderadamente espesa le permitió al paciente un manejo lento, pausado del alimento en boca. Además, permitió al proceso la inhibición de riesgos al sesgar al mínimo el control que la gravedad ejerce sobre el alimento, adaptando la postura y los volúmenes que se proporcionaban. Inicialmente se suministraba la alimentación a través de cucharas con una cantidad de $5 \mathrm{ml}$. Cantidad que se aumentó en un mes de terapia a $10 \mathrm{ml}$, fundamentado a partir de acciones de aumento propioceptivo, alcance de movimiento, fuerza y resistencia muscular.

Al observar mayor sensibilidad a nivel posterior intraoral y mejor control, fuerza y coordinación de lengua, se inicia el suministro de la consistencia nivel 2, poco espesa, con la cual se provee de un estímulo a nivel sensorial intraoral diferente, incluyendo el pitillo como elemento para ofertar alimento, permitiendo involucrar en mayor porcentaje el grupo muscular labial al proceso alimenticio.

Los avances en los tiempos de discriminación de las texturas, temperatura y manejo de la cantidad del bolo en cavidad oral, al igual que de desencadenamiento del reflejo deglutorio con la propulsión a fase orofaríngea, permitieron el aumento de la ingesta por vía oral en una proporción inicial de $\mathbf{3 0} \%$ vía oral - $\mathbf{7 0} \%$ vía enteral a un $\mathbf{1 0 0 \%}$ incluyendo a la dieta el nivel 4-puré, según la IDDSI.

Para el proceso de intervención, se incluye a la familia y cuidadores como parte del equipo de apoyo terapéutico, implementando estrategias que permitan su participación responsable en la rehabilita-

ción, favoreciendo un espacio seguro para la comunicación y la ingesta del alimento (Notario, Arauz, Granados, \& Marmol, 2011).

(Arroyo-Aljaro, González-Viejo, \& Rovira, 2008), menciona que existe una clasificación para la recuperación funcional del paciente con LIS; hace referencia a 5 niveles descritos a continuación:

Sin recuperación: pacientes sin recuperación motora y totalmente dependientes para su cuidado.

Mínima recuperación: pacientes que presentaron alguna mínima recuperación motora voluntaria, pero totalmente dependientes en su cuidado.

Moderada recuperación: pacientes que tuvieron una recuperación significativa, permitiéndoles independencia en alguna de las actividades de la vida diaria, no en todas.

- Total recuperación: pacientes que consiguieron independencia en todas las actividades de la vida diaria, pero con mínimo déficit neurológico.

Pacientes sin déficit neurológico. El caso desarrollado en este artículo se encuentra en un nivel 2 de recuperación funcional.

\section{Consideraciones éticas}

El caso cumple con el propósito principal de la investigación médica en seres humanos, buscando comprender las causas, evolución y efectos del síndrome, además de fundamentar procesos preventivos, diagnósticos y terapéuticos positivos para la evolución del caso. (World Medical Assault Strength, 2013).

Las acciones desarrolladas fueron sujetas a normas éticas contempladas en la República de Colombia, promoviendo y asegurando el respeto a los seres humanos, su salud, privacidad y derechos individuales. En este orden de ideas, la atención clínica suministrada y el registro del caso fueron regidos por normas científicas, técnicas y administrativas para la investigación en salud descritas en el país a partir de los lineamientos de la Resolución 8430 (República de Colombia. Ministerio de Salud, 1993)

Partiendo de lineamientos éticos, se establece el consentimiento informado de los padres y posteriormente de la paciente como requisito indispensable para la ejecución de las acciones terapéuticas.

Las acciones de proceso deglutorio, se enmarcan según la resolución 8430, como procedimientos de riesgo mínimo, interpretados como la posibilidad de penetración y aspiración propios de la acción alimenticia, resultados de la restricción motora inherente al síndrome, coadyuvado por la estimulación intraoral permanente que se ejecuta en terapia, aunque con una proporción riesgo-beneficio favorable, dado que estos riesgos deben ser transitados en la rehabilitación para impactar con el tratamiento en acciones deglutorias eficaces y seguras.

\section{Discusión}

Es necesario visualizar la disfagia como un complejo de síntomas que puede causar la muerte del paciente, por lo tanto, la acción terapéutica que intervenga en el manejo, control y reorganización de los procesos neurofisiológicos de la alteración deglutoria, será estrictamente necesaria.

Como una consecuencia neurogénica, la disfagia es el efecto de una desconexión neural, que en la afección de la respuesta motora 
espontánea del paciente con LIS se convierte en un factor altamente restrictivo de las acciones orales y faríngeas que se establecen como voluntarias e involuntarias; sin embargo, como menciona (Vidal, 2018), a pesar del estado de mínima respuesta ante una instrucción de movimiento facial, se puede presentar respuestas emocionales con relevancia ambiental que lleguen a generar respuestas motoras no atribuidas a actividad refleja.

En los pacientes diagnosticados con LIS, la causa principal de la alteración deglutoria proviene de una desconexión pontina que altera la cara anterior de la protuberancia, influyendo en la interrupción de los tractos corticoespinales y corticobulbares comprometiendo el suministro a núcleos motores medulares que apoyan, entre otras, a la deglución.

La fase oral es considerada, una fase semi automática, que actúa bajo un comando subcortical, capaz de responder las actividades programadas en ausencia del comando cortical. El tronco cerebral es un centro responsable por la actividad refleja de la fase faríngea (Mansueto, Aguiar, Oliveira, Caseiro, \& Teixeira, 2016), así como de la musculatura estriada de la porción superior del esófago. Todos los sub-núcleos se relacionan con actividades corticales y subcortical, son estos, puntos de sinapsis para las vías aferentes y eferentes, alteradas en el LIS.

Los nervios craneanos eferentes (nervio facial-VII, nervio trigémino $V$ en la rama mandibular-V3, nervio hipoglóso-XII, nervio vago-X), son responsables por la dinámica de las fases oral y faríngea de la deglución. Los segmentos del plexo cervical C1 hasta C3 tiene una interferencia con la dinámica neural faringoesofágica de la deglución. Todo eso define a la deglución como un proceso funcional multicéntrico (Costa, 2013), que al alterarse en todo o cualquier punto de la secuencia y debido a la desconexión pontina, genera en el LIS un descontrol deglutorio.

Dadas las características multicéntricas, el funcionamiento de los centros encargados de la secuencialidad de los procesos neurofisiológicos de la deglución juegan un papel importante y deben ser establecidos como el centro fundamental de la intervención fonoaudiológica para minimizar los riesgos, objetivo que se planteó desde una coordinación ideal en el transporte del bolo alimenticio en boca y faringe, principalmente. Por esta razón se adoptó como estrategia fundamental el manejo intraoral de las consistencias, como fortalecimiento sensorial, motor y de manejo del riesgo. Por ello se usó el sabor ácido y temperatura fría.

Cabe destacar que en individuos con secuelas neurológicas el tiempo de tránsito orofaríngeo es menor, cuando el bolo con sabor ácido es deglutido (Cola, Gatto, Gonçalves, Schelp, \& Coelho, 2008). Ya que, las vías sensoriales del sabor cuando son estimuladas con sabores fuertes, como el ácido, puede servir como un estímulo de alerta para el tronco cerebral y centros corticales de la deglución, explicando así los cambios significativos en las medidas de tiempo con relación a las fases de la deglución. En esta perspectiva, la temperatura fría y el sabor ácido alteran el comportamiento de la biomecánica deglutoria en personas saludables y en pacientes neurológicos.

El establecimiento de las estrategias para aumentar el input sensorial en función a los factores exógenos (volumen, consistencias, temperaturas) que engloban la deglución y promueven la acción alimenticia se adoptaron para generar cambios neurofuncionales que activarán en mayor medida los comandos neurales de la deglución, apropiando la secuencia valvular de la deglución y aminorando el riesgo de aspiración; por acción sensitiva, los comandos motores de la deglución alteran su dinámica, estableciendo en función a la actividad sensorial ofertada por la consistencia, los sabores y las temperaturas, una acción secuencial motora adaptada a las necesidades y los riesgos (Ney, Weiss, Kind, \& Robbins, 2009) (Steele, y otros, 2015).

Según (Costa, 2013), la deglución es un proceso neuromotor complejo, que se basa en eventos de despolarización muscular y actividades sensoperceptuales de índole semiautomáticas (voluntarias e involuntarias) y automáticas (involuntarias). La fase oral de la deglución tiene características fisiológicas voluntarias (captación del bolo, masticación, lateralización del bolo, eyección) y características fisiológicas involuntarias sensoriales mediadas por el Input sensorial (percepción táctil, percepción gustativa, salivación). En la fase faríngea (involuntaria), incluye el desencadenamiento de reflejos protectores de la deglución (gag, tos y disparo de la deglución); hasta contracciones musculares coordinadas a nivel laríngeo y faríngeo, que permiten la elevación y anteriorización del sistema hiolaríngeo, que a su vez propician la protección de las vías aéreas inferiores por medio del selle de la laringe en el tránsito faríngeo del bolo alimenticio, todo como efecto de acciones sensoriales (Clavé, y otros, 2008) (Vargas M. , 2015).

\section{Conclusiones}

Dado el pronóstico reservado a nivel médico para los casos con LIS, donde el compromiso neurológico no favorece los procesos sensoriales y motores de quien lo presenta, la intervención fonoaudiológica oportuna y estructurada desde las necesidades sensoriomotoras en relación a su descripción clínica de base, permitió en este caso una evolución con resultados positivos a 6 meses, donde las acciones encaminadas al favorecimiento de procesos sensoriales y motores orales en pro de la alimentación, han dado paso a habilidades motoras orofaciales que sustentan la habilitación de la ingesta de alimento $100 \%$ por vía oral, usando modificaciones de consistencias.

La experiencia en el caso aporta como fundamento estratégico la utilización de consistencias como herramienta en la terapéutica, especificando la importancia que se debe plantear igualmente proceso de acompañamiento y suministro de la alimentación por parte del cuidador principal, que es en últimas en quien recae la responsabilidad de proporcionar de manera correcta el alimento.

La estrategia de consistencia como utilización de inhibición de riesgo no se debe plantear de manera aislada; con ella merece la pena definir el volumen de la ingesta, los tiempos de proporción del alimento, el aditamento que se utilice y la postura que se adopte para el momento de la alimentación.

\section{Bibliografía}

Alvarez, C., Llorente, J., Gutierrez, V., Fernandez, H., Guervos, M., Suarez, C., \& Hermen, M. (2007). Recidivas en el cáncer escamoso de cabeza y cuello. Acta Otorrinolaringol Esp, 58(4), 156-163.

American Cancer Society, NHC. (2015). Recuperado el 5 de Octubre de 2016, de Una guía sobre Radioterapia: http://www.cancer.org/acs/ groups/cid/documents/webcontent/003027-pdf.pdf

American Cancer Society, NHC. (2015). American Cancer Society. Recuperado el 5 de Octubre de 2016, de Guía sobre Radioterapia: https://www.cancer.org/es/tratamiento/tratamientos-y-efectossecundarios/tipos-de-tratamiento/radioterapia.html

Arroyo-Aljaro, R., González-Viejo, M., \& Rovira, A. (2008). Síndrome de locked-in. Una mirada a su tratamiento y pronóstico. Rehabilitación, 42(2), 92-95. doi:https://doi.org/10.1016/S0048-7120(08)73620-X 
Abordaje fonoaudiológico de la deglución en el Síndrome de Enclaustramiento

Informe de un caso

Barros, A., Silva, S., \& De Angelis, E. (2010). Videofluoroscopia da deglutição orofaríngea. En G. Jotz, E. Carrara-De-Angelis, \& A. Barros, Tratado de deglutição e disfagia: no adulto e na criança. (págs. 84-88). Rio de Janeiro: Revinter.

Belafsky, P., Blumenfeld, L., LePage, A., \& Nahrstedt, K. (Nov de 2003). The accuracy of the modified Evan's Blue Dye Test in predigting aspiration. Laryngoscope, 113(11), 1969-72. doi :c10.1097/00005537-200311000-00021

Biblioteca Virtual de Salud. (15 de Mayo de 2018). Descriptores en Ciencias de la Salud. Obtenido de DeCS: http://decs.bvs.br/cgi-bin/wxis1660. exe/decsserver/

Cadena, E., Guerra, R., \& Pérez-Mitchell, C. (Julio-Septiembre de 2014). Cirugía Robótica Transoral (TORS), en el manejo de lesiones neoplásicas de cabeza y cuello. Revista Colombiana de Cancerología, 18(3), 128-136. doi:10.1016/j.rccan.2014.06.003

Cámpora, H., \& Falduti, A. (2012). Evaluación y tratamiento de las alteraciones de la deglución. Rev Am Med Resp, 3(1), 98-107.

Cárcamo. (2018). Epidemiología y generalidades del tumor de cabeza y cuello. Revista Médica Clínica Las Condes, 29(4), 387-492. doi:10.1016/j.rmclc.2018.06.009

Carcamo, M. (Ago de 2018). Epidemiología y generalidades del tumor de cabeza y cuello. Revista Médica Clínica Las Condes, 29(4), 387-492.

Clavé, P., Arreola, V., Romea, M., Medina, L., Palomera, E., \& Serra-Prat, M. (2008). Accuracy of the volume-viscosity swallow test for clinical screeneng of oropharyngeal dysphagia and aspiration. Clinical Nutrition, 2(7), 806-15. doi:https://doi.org/10.1016/..clnu.2008.06.011

Cola, P., Gatto, A., Gonçalves, R., Schelp, A., \& Coelho, M. (2008). Reabilitação em disfagia orofaríngea neurogênica: sabor azedo e temperatura fría. Rev. CEFAC, 10(2). doi:https://doi.org/10.1590/S151618462008000200009

Costa, M. (2013). Controle Neural da Deglutição. En Deglutição \& Disfagia: Bases Morfofuncionais e Videofluroscópicas (págs. 51-53). Rio de Janeiro: Medbook

Denaro, N., Merlano, M., \& Russi, E. (Sep de 2013). Dysphagia in Head and Neck Cancer Patients: Pretreatment Evaluation, Predictive Factors, and Assessment during Radio-Chemotherapy, Recommendations. Clinical and Experimental Otorhinolaryngology., 6(3), 117126. doi:https://www.e-ceo.org/journal/view.php?doi=10.3342/ ceo.2013.6.3.117

European Society for Medical Oncology. (01 de 01 de 2015). Fundación contra el cáncer. Recuperado el 18 de 06 de 2019, de ESMO: ESMOACF-Cancer-de-Cabesa-y-Cuello-Guia-para-pacientes.pdf

Fiorelly, A., Fausto, F., Nagar, F., Fusco, P., Mazzone, S., Costa, G., .. Santini, M. (31 de abril de 2017). A New Modified Evans Blue Dye Test as Screening Test for Aspiration in Tracheostomized Patients. Journal of cardiothoracic and vascular anesthesia., 2, 441-445. doi:https:// doi.org/10.1053/j.jvca.2016.07.031

Flores, K., Berretin-Felix, G., Beltrati, M., \& Queiroz, I. (ABRIL-JUNIO de 2009). Avaliação miofuncional orofacial - Protocolo MBGR. CEFAC, 11(2), 237-255.

Frowen, J., Hughes, R., \& Skeat, J. (2019). The prevalence of patientreported dysphagia and oral complications in cancer patients. Supportive Care in Cancer, doi: 10.1007/s00520-019-04921-y.

Furkim, A., \& Freitas, A. (2008). Eficácia da fonoterapia em disfagia neurogênica usando a escala funcional de ingestão porvia oral (FOIS) como marcador. Rev CEFAC, 10(4), 503-512. doi:https://doi. org/10.1590/S1516-18462008000400010

Greenblatt, D., Sippel, R., Leverson, G., Frydman, F., Schaefer, S., \& Chen, H. (2009). Thyroid Resection Improves Perception of Swallowing Function. World Journal of Surgery, 33, 255-260. doi:10.1007/s00268008-9837-9

Guevara, E., Avendaño, S., Salazar, A., Alarcón, M., \& Santelices, M. (2016). Contribución de la temperatura fría y el sabor ácido en la intervención fonoaudiológica de la disfagia orofaríngea. Revista chilena de fonoaudiología, 15, 1-9. doi:10.5354/0719-4692.2016.44206

Hamilton, S., Yoo, J., Hammond, A., Read, N., Venkatesan, V., Franklin, J., . Badhwar, A. (Oct de 2008). Microvascular changes in radiationinduced oral mucositis. J Otolaryngol Head Neck Surg, 37(5), 730737.

Hutcheson, K., Lewin, J., Barringer, D., Lisec, A., Gunn, B., Moore, M., \& Holsinger, C. (2012). Late Dysphagia After Radiotherapy-Based Treatment of Head and Neck Cancer. Cancer, 118(23), doi: 10.1002/ cncr.27631.

International Dysphagia Diet Standardisation Initiative. (10 de October de 2016). Complete IDDSI framework and descriptors. Obtenido de iddsi.org/translation: https://iddsi.org/wp-content/uploads/2018/05/ Marco y descriptores de la IDDSI FOR REVIEW.pdf

International Dysphagia Diet Standardisation Initiative. (01 de Agosto de 2018). International Dysphagia Diet Standardisation Initiative. Obtenido de IDDSI: http://iddsi.org/

Joung, S., Woo, K., Beom, S., Hwa, J., \& Kyu, M. (Oct de 2015). Voluntary Cough and Swallowing Function Characteristics of Acute Stroke Patients Based on Lesion Type. Archives of Physical Medicine and Rehabilitation, 96(10), 1866-1872. doi:https://doi.org/10.1016/j. apmr.2015.06.015

Khanna, K., Verma, A., \& Richard, B. (December de 2011). The locked-in syndrome: Can it be unlocked? Journal of Clinical Gerontology and Geriatrics, 2(4), 96-99.

Lazarus, C. (Jun de 2009). Effects of chemoradiotherapy on voice and swallowing. Curr Opin Otolaryngol Head Neck Surg, 17, 172-178.

Lazarus, C., Ward, E., \& Yiu, E. (2014). Speech and Swallowing following oral, oropharyngeal and nasopharyngeal cancers. En E. Ward, \& C. Van As-Brooks, Head and Neck Cancer: Treatment, Rehabilitation, and Outcomes (págs. 173-240). San Diego, California: Plural Publishing.

Liga contra el cáncer. (01 de 01 de 2016). Liga contra el cáncer seccional Bogotá. Recuperado el 18 de 06 de 2019, de http://www. ligacontraelcancer.com.co/cancer-de-cabeza-y-cuello/

Liga Contra el Cáncer. (01 de 01 de 2016). Liga contra el cáncer seccional Bogotá. Recuperado el 18 de 06 de 2019, de http://wwW. ligacontraelcancer.com.co/cancer-de-cabeza-y-cuello/

Logemann, J., Pauloski, B., Rademaker, A., \& Colangelo, L. (1 de Mayo de 1997). Speech and swallowing rehabilitation for head and neck cancer patients. Oncology (Williston Park), 11(5), 659-664.

Lujan Ramos, V. P., Monterrosa-Salazar, E., \& Polo-Verbel, L. (2011). Síndrome de enclaustramiento: a propósito de un caso. Revista Ciencias Biomédicas, 2(1), 116-120.

Lyndell, K. (2014). Radiation and Chemotherapy. En E. Ward, \& C. Van as-brooks, Head and neck cancer: treatment, rehabilitation, and outcomes (Second Edition ed., págs. 63-102). San Diego, California: Plural Publishing.

Mansueto, A., Aguiar, S., Oliveira, e., Caseiro, L., \& Teixeira, A. (2016). Frequência e fatores associado à disfagia após acidente vascular cerebral. CoDAS, 28(1), 66-70. doi:https://doi.org/10.1590/2317$1782 / 20162015072$

Martínez, M., \& Bellido, D. (2003). Nutrición y disfagia. Fisioterapia, 25(5), 306-310. doi:https://doi.org/10.1016/S0211-5638/03)73069-7

Ney, D., Weiss, J., Kind, A., \& Robbins, J. (2009). Senescent Swallowing: Impact, Strategies and Interventions. Nutr Clin Pract., 24(3), 395-413. doi:doi:10.1177/0884533609332005

Nickel, B., Tan, T., Cvejic, E., Baade, P., McLeod, D., Pandeya, N., ... Jordan, S. (17 de 01 de 2019). Health-Related Quality of Life After Diagnosis and Treatment of Differentiated Thyroid Cancer and Association With Type of Surgical Treatment. JAMA Otolaryngology-Head \& Neck Surgery, e1-e8. doi:10.1001/jamaoto.2018.3870.

Nickel, B., Tan, T., Cvejic, E., Baade, P., McLeod, D., Pandeya, N., . . Jordan, S. (17 de jan de 2019). Health-Related Quality of Life After Diagnosis and Treatment of Differentiated Thyroid Cancer and Association With Type of Surgical Treatment. JAMA Otorinolaryngology - Head \& Neck Surgery, 231-238. doi:10.1001/jamaoto.2018.3870 
Notario, M., Arauz, L., Granados, F., \& Marmol, P. (22 de Febrero de 2011). Plan de cuidados en el síndrome de cautiverio. Enfermería Clínica, 21(2), 104-109. doi:https://doi.org/10.1016/j.enfcli.2010.11.005

O'Neil, K., Purdy, M., Falk, J., \& Gallo, L. (1999). The dysphagia outcome and severity scale. Dysphagia., 14(3), 139-145. doi:https://doi.org/10.1007/ PL00009595

Orphadata. (17 de Mayo de 2018). Clasifications of rare diseases: hierarchical representation. Obtenido de http://www.orphadata.org/ cgi-bin/inc/product3.inc.php

Pacheco-Hernández, A., Ramos Villegas, Y., Padilla Zambrano, H., Mendoza Florez, R., Quintana Pájaro, L., López Cepeda, D., ... Moscote Salazar, L. R. (2017). Síndrome de Locked-In: Reporte de Caso. Revista Ecuatoriana de Neurología, 26(3), 301-305.

Pauloski, B. (Nov de 2008). Rehabilitation of Dysphagia Following Head and Neck Cancer. Physical Medicine and Rehabilitation Clinic of North America, 19(4), 889-928. doi: 10.1016/j.pmr.2008.05.010.

Pereira, G., Carrera, E., \& Brandão. (2010). Principios da reabilitação das disfagias orofaríngeas. En G. Jotz, E. Carrara-De Angelis, \& A. Barros, ratado da deglutição e disfagia No adulto e na criança (págs. 332339). REVINTER

Portas, J., Pereira, C., Perissato, E., Dos Santos, D., Sampaio, A., Aparecido, R., \& Brandao, A. (Ene-Feb de 2011). Swallowing after non-surgical treatment (radiation therapy / radiochemotherapy protocol) of laryngeal cancer. Brazilian Journal of Otorhinolaryngology, 77(1), 96-101.

República de Colombia. Ministerio de Salud. (1993). Resolución $N^{\circ}$ 008430. Bogotá: Ministerio de Salud, Rama ejecutiva - República de Colombia.

Rosenbek, J., Robbins, J., Roecker, E., Coyle, J., \& Wood, J. (1996). A penetration-aspiration scale. Dysphagia, 11(2), 93-98. doi:https://doi. org/10.1007/bf00417897

Santini, L., Robert, D., Lagier, A., Dessi, P., \& Fakhry, N. (Junio de 2015). A videofluoroscopic study comparing severe swallowing disorders in patients treated surgically or with radiation for oropharyngeal cancer. International Journal of Oral and Maxillofacial Surgery, 44(6), 705709. doi:https://www.ijoms.com/article/S0901-5027(15)00044-2/fulltext

Shaw, S., \& Martino, R. (Dec de 2013). The normal swallow: muscular and neurophysiological control. Otolaryngology Clinics of North America, 46(6), 937-956. doi:10.1016/j.otc.2013.09.006.

Simao, M., Alacid, C., Rordrigues, K., Albuquerque, C, \& Furkim, A. (Oct-Dec de 2009). Incidence of tracheal aspiration in tracheotomized patients in use of mechanical ventilation. Arquivos de gastroenterologia, 46(4), 311-314. doi:10.1590/50004-28032009000400012

Siu, L., Waldron, J., Chen, B., Winquist, E., Wright, J., Nabid, A., .. J Johnson, A. (Dic de 2016). Effect of Standard Radiotherapy With Cisplatin vs Accelerated Radiotherapy With Panitumumab in Locoregionally Advanced Squamous Cell Head and Neck Carcinoma: A Randomized Clinical Trial. JAMA oncology, 3(2), 220-226.
Smeele, L. (2014). Oral, oropharyngeal and nasopharyngeal cancer: intervention approaches. En E. Ward, \& C. Van As-Brooks, Head and neck cancer: treatment, rehabilitation, and outcomes ( ed., págs. 103-120). San Diego, California: Plural Publishing.

Steele, C., Abdulrahman, W., Ayanikalath, S., Barbon, C., Chen, J., Cichero, J., ... Wang, H. (Octubre de 2015). The Influence of Food Texture and Liquid Consistency Modification on Swallowing Physiology and Function: A Systematic Review. Dysphagia, 30, 2-26. DOI 10.1007/ s00455-014-9578-x

Suárez, L., Puerto-García, S., Rodríguez-Moreno, L., \& Ramírez-Moreno, J. (2017). La crisis del sistema de salud colombiano: una aproximación desde la legitimidad y la regulación. Rev. Gerenc. Polit. Salud, Bogotá, Colombia, 16(32), 34-50.

Szczesniak, M., Maclean, J., Zhang, T., Graham, P., \& Cook, I. (Nov de 2014). Persistent Dysphagia after Head and Neck Radiotherapy: A Common and Under-reported Complication with Significant Effect on Noncancer-related Mortality. Clinical Oncology (Royal College of Radiologist), 26(11), 697-703. doi: 10.1016/j.clon.2014.08.009

Vargas, M. (2015). Algoritmo de transformación de consistencia como estrategia de abordaje fonoaudiológico. . Rev. Col. Reh., 14(1), 22 - 27.

Vargas, M. (30 de junio de 2017). Repercusiones sociales de la disfagia: revisión sistemática. Revista Colombiana de Rehabilitación, 16(1), 32-39. doi:https://doi.org/10.30788/RevColReh.v16.n1.2017.63

Vargas, M. (Abril-Junio de 2018). Repercusiones sociales de la disfagia. Revista de Logopedia, Foniatría y Audiología, 38(2), 77-83. doi:https://doi.org/10.1016/j.rlfa.2017.10.003

Vidal, F. (2018). Hacia una fenomenología del síndrome de cautiverio. Rev. Asoc. Esp. Neuropsiq., 38(133), 45-73. doi:10.4321/S021157352018000100003

Wheeler-Hegland, K., Ashford, J., Frymark, T., McCabe, D., Mullen, R., Musson, N., . Schooling, T. (2009). Evidence-based systematic review: Oropharyngeal dysphagia behavioral treatments. Part II-Impact of dysphagia treatment on normal swallow function. Journal of rehabilitation research and development, 46(2), 185-194.

World Medical Assault Strength. (2013). 64th General Assembly. Declaration of Helsinki of the amm - Ethical Principles for Medical Investigations in Human Beings. Fortaleza: World Medical Association. Obtenido de https://www.wma.net/policies-post// wma-declaration-of-helsinki-ethical-principles-for-medical-researchinvolving-human-subjects/.

Zhang, L., Huang, Z., Wu, H., Chen, W., \& Huang, Z. (Dic de 2014). Effect of swallowing training on dysphagia and depression in postoperative tongue cancer patients. Eur J Oncol Nurs, 18(6), 626-629. 